\author{
Aníbal Gauna \\ Sumiko Burga \\ Renato Córdoba \\ Alexandra Martínez \\ Valeria Pariahuachi \\ Fabricio Polar
}

Escuela de Comunicación y Periodismo.Universidad Peruana de Ciencias Aplicadas eagauna@fulbrightmail.org/sumikoburgaortiz.15@gmail.com / cordovarenato2o18@gmail.com /

lasallealexandra@gmail.com / pr.nallelyvaleria@gmail.com / fabriciopolarc@gmail.com

\title{
DETERMinando la “MORALIDAD PÚBLICA” DE LAS MUJERES. UNA PERSPECTIVA RELACIONAL SOBRE EL ACOSO SEXUAL CALLEJERO EN ESTUdiantes UNIVERSiTARIOS DE LIMA, PERÚ
}

\begin{abstract}
Resumen: Con un enfoque relacional y sobre la base de 21 entrevistas semiestructuradas a estudiantes universitarios en Lima (Perú), este artículo describe una dimensión poco explorada del acoso sexual callejero (ASC): su uso como una forma derivada de determinar la moralidad de las mujeres en el espacio público. Al hacerlo articulamos el enigma de la práctica del ASC (su inconsecuencia para fines prácticos sexuales) con su puesta en escena para otros hombres (práctica homo-social), para sí mismos como hombres, y con el nivel más general de la dominación masculina sobre las feminidades.
\end{abstract}

Palabras clave: Sociología relacional, Dominación masculina, Acoso sexual callejero

Determining women's "public morality". A relational perspective on street sexual harassment amonh university students in Lima, Peru

\footnotetext{
Abstract: With a relational approach and on the basis of on 21 semi-structured interviews with university students in Lima (Peru), this article describes a little-explored dimension of street sexual harassment (SSH): its use as a derivative way of determining the morality of women in the public space. In doing so we articulate the enigma of SSH (its inconsistency for practical sexual purposes) with its staging for other men (homo-social practice), for themselves as men, and with the more general level of male dominance over femininities.
}

Keywords: relational sociology, male dominance, street sexual harassment. 


\section{Introducción}

En la última década han proliferado los estudios en los cuales se argumenta que en la violencia de género cabe incluir una antigua y aparentemente inocua práctica como es el acoso sexual callejero (Dhillon y Bakaya, 2014; Flores, 2019; Martínez, 2018; Sánchez-Díaz, 2019; Vallone y Quiroga, 2019; Wesselman y Kelly, 2010) -en adelante ASC. En realidad, este no existía hasta hace relativamente muy poco tiempo, pues era prácticamente invisible en un doble sentido: primero como toda violencia contra la mujer, pero también porque no era epistémicamente tangible. Por supuesto, sobre todo las mujeres y las minorías sexuales la conocían muy bien al padecerla, pero era una cuestión normalizada y minimizada, que pasaba desapercibida. Dehecho, según Di Leonardo (1981), quizá la primera investigadora en usar la expresión acoso callejero (street harassment), hace apenas cuarenta años se veía como un aspecto trivial en comparación con la violencia en la esfera privada, en acciones tales como las violaciones, las golpizas o el acoso sexual en el lugar de trabajo. Pero hoy en día se considera que el ASC es un eslabón en la cadena de violencia contra las feminidades y las personas asociadas con estas, a veces un preámbulo a la violencia física severa (Logan, 2015), e incluso una violación de los derechos humanos que reduce la viabilidad económica y social de las ciudades (Fraser, Kalpana y MacLean, 2017). El ASC es facilitado por la comunicación en espacios públicos y se define como la experiencia de la atención sexual no deseada por parte de extraños en dichos espacios (Wesselmann y Kelly, 2010). El mismo ha sido clasificado en cinco dimensiones o formas: verbal, expresiva, física, persecuciones y exhibicionismo (Gaytán, 2007), formas que incluyen actos tales como pellizcar, dar nalgadas, golpear, gritar a distancia, gestos o palabras vulgares, insultos, insinuaciones, ruidos de besos, comentarios maliciosos, silbidos, miradas lascivas, masturbación pública, toqueteos y acechamiento (Gardner, 1995; Rodríguez, 2020; Vallejo y Rivarola, 2013). Su consecuencia más evidente es la invasión del espacio personal, en este caso, de las mujeres.

Apenas fue el 20 de diciembre de 1993, cuando la ONU definey hace patente de manera formal el ASC en la Declaración sobre la eliminación de la violencia contra la mujer. Previamente se había considerado que el mismo era algo tan común en la vida pública que incluso las mismas mujeres se convencieron 
de que nadie pensaría que algo de suficiente significancia habría ocurrido si acaso habían sido víctimas de ASC, pues el mismo había sido trivializado (Chafai, 2020). Así es que para poder identificar al ASC como una forma de violencia, como mínimo dos cambios han tenido que darse. Para empezar, ha ocurrido una ampliación de la consideración de aquello que la violencia de género ha incluido, cosas tales como gestos físicos y verbales, ampliación que ha sido estudiada principalmente desde el punto de vista de las consecuencias que tiene para la subjetividad de las víctimas (Davidson, Butchko, Robbins, Sherd, and Gervais, 2016; Sánchez-Díaz, 2019). Estas consecuencias incluyen la sensación de pérdida de control, la disminución de la autoestima, distorsiones en la valoración cognoscitivas de las experiencias de acoso y un incremento en la inseguridad propia y en la desconfianza hacia hombres desconocidos (Gaytán, 2007).

Por otra parte, se ha ampliado el espectro del espacio social de análisis de la violencia de género, pasando a considerar no solo la violencia simbólica que se comete en el ámbito de lo privado sino también aquella que ocurre en el ámbito público (Flores, 2019). Esta conjunción que se ha dado entre el reconocimiento de nuevas técnicas micro-corporales de agresión y nuevos espacios sociales (públicos), abre la puerta para una consideración performativa del fenómeno del ASC (Quinn, 2002). Si bien en este artículo no analizamos toda la puesta en escena de tales performances, sí buscamos dar cuenta de los significados que dicha escenificación tiene para los sujetos acosadores. Como lo argumentaremos sobre la base de veintiún entrevistas semi estructuradas (quince a hombres jóvenes que han cometido ASC y seis a mujeres jóvenes que han sufrido ASC), y de manera consistente con previos estudios, el ASC viene a ser parte de una matriz de dominación simbólica, cuyo carácter performativo es de triple faz. Por un lado, ciertamente se busca ejercer una forma de dominio sobre el género femenino, o sobre otras sexualidades alternativas (nos limitaremos acá a las relaciones de acoso de hombre a mujer, como la forma más generalizada entre nuestra población de estudio, jóvenes universitarios). Pero, segunda faceta, al momento de explicar la permanencia y generalidad del ASC en particular, quizá lo más relevante sea que al menos inicialmente es una puesta en escena de la masculinidad frente a otros hombresy frente a sí mismos como hombres (Molina, López y Chávez, 2020; Quinn, 2002). La 
tercera faceta, como un efecto derivado de los dos anteriores, muestra que el ASC funciona también para los acosadores distinguir entre las mujeres "buenas" y las "malas" mujeres, consistente con la distinción machista entre la mujer "santa" y la mujer "puta". Al considerarlo, exponemos un significado que no ha sido mencionado en la literatura sobre el ASC: el mismo funciona también para mostrar en público la supuesta 'moral' de las mujeres acosadas. Con esto no queremos decir que estos cuasi-rituales simplemente expresen o reflejen un significado del género, sino que son dicho significado de manera específicamente relacional, es el género-sociedad, en tres estructuras de relaciones públicas: hombre-mujer, hombre-hombre y sí-mismo como hombre o como mujer. Describir estos significados y su funcionamiento desde la perspectiva de los sujetos acosadores, sobre los que sigue existiendo mucha menos investigación hasta recientemente (Callirgos 1998; Molina, López y Chávez, 2020), es el objetivo del presente artículo.

\section{El ASC en general y en Lima}

ElASC es un fenómeno global del cual hay registro al menos desde los inicios del siglo XIX (Heben, 1994, citado por Logan, 2015, p. 201), y, aunque actualmente las estadísticas confiables y comparable sobre el mismo son escasas o inexistentes (Fraser, Kalpana y MacLean, 2017), una aproximación general nos da una idea de su extensión. Para el año 1995, 100 por ciento de mujeres entrevistadas en una muestra racialmente diversa de 293 mujeres en los EE. UU. reportaba haber sido acosada alguna vez (Gardner, 1995, citada por Logan, 2015, p. 201). Dos estudios en Canadá mostraron que, en los noventa, de una muestra de 12.300 mujeres 85 por ciento habían sido acosadas en la calle de alguna manera (MacMillan et al, 2000, citada por Logan, 2015, p.201). De acuerdo con Fraser, Kalpana y MacLean (2017, p.10) sobre la base de distintos informes, el ASC llegaría en el 2010: al $68 \%$ en Ecuador; en Londres (Inglaterra) al 75\%, en Tailanda al 86\% y en Brasil al 89\% según informe del 2016 (con N de mínimo 380 para cada ciudad en el estudio), mientras que en Puerto Moresby (Papúa Nueva Guinea) alrededor del 90\% de mujeres han sufrido alguna forma de violencia sexual en el transporte público. En países como Marruecos, aunque el ASC callejero ha sido poco estudiado, cifras oficiales del Ministerio de Familia, Solidaridad, Equidad y Desarrollo Social estiman que sobre el 
12\% han sufrido violencia en el espacio público (Chafai, 2020, 4). Testimonios de mujeres de Delhi, India, indican que han sufrido acoso callejero hasta en un 50\% de sus visitas a lugares públicos (Dhillon y Bakaya, 2014, p.4). Según un estudio realizado en el 2018, de una muestra de 6.205 mujeres mayores de 18 años I de cada 3 mujeres en España ha sufrido algún tipo de ASC (Rodríguez, 2020, p.4). En 2014, una encuesta de 6,555 mujeres y expertos en las 16 capitales más grandes del mundo (de acuerdo con su volumen poblacional), encontró que 6 de cada 10 mujeres en capitales latinoamericanas habrían sido acosadas en el transporte público (Thomas Reuters Foundation y YouGov, citada por Fraser, Kalpana y MacLean, 2017, p.9).

En otro estudio en la Provincia de Buenos Aires, Argentina, basado en un estudio nacional más amplio de 86.900 casos, se encontró que sobre el $94 \%$ había recibido alguna vez comentarios sobre su apariencia o un toque de bocina o claxon (estudio del Observatorio de Género del Instituto de Proyección Ciudadana, citado por Vallone y Quiroga, 2019, p.2). A pesar de estas elevadas cifras, como lo muestran Dhillon y Bakaya (2014), y Fraser, Kalpana y MacLean (2017), el ASC se encuentra subrepresentado en las estadísticas oficiales porque las víctimas lo denuncian poco, además de estar poco atendido por políticas oficiales. Esto se debe en buena medida a que las mujeres encuentran que sus denuncias son ignoradas o al menos no atendidas con suficiente eficacia.

Perú no es excepcional en este sentido. Por el contrario, según una encuesta del año 2017, la ciudad de Lima ocupó el quinto puesto entre las diez megaciudades del mundo con mayor presencia de acoso y violencia sexual (Thomson Reuters Foundation 2017, citada por Alcalde 2020, p.25). En particular, la sensación de inseguridad al transitar por la calle es desigual entre hombres y mujeres en todo el país, lo cual constituye una barrera invisible para el libre tránsito de las mujeres por las calles (Vallejo y Rivarola, 2013). Mujeres que han retornado a Lima luego de vivir en el extranjero también perciben que en la ciudad el acoso callejero es muy agresivo (Alcalde, 2020). Más específicamente aún, según cifras del Observatorio de Criminalidad del Ministerio Público del Perú, el 82.4\% de los delitos de violación de la libertad sexual a nivel nacional se registraron en Lima y Callao (Vallejo y Rivarola, 2013, p.4). Por todo esto, el fenómeno del ASC en la ciudad de Lima merece una atención especial. 


\section{Diseño de la investigación}

Se realizaron 15 entrevistas semiestructuradas a varones y 6 a mujeres jóvenes de entre 18 y 22 años, estudiantes universitarios, de diversas características raciales, residentes en la ciudad de Lima. La selección de las y los entrevistadas(os) fue por bola de nieve a través de estudiantes de la carrera de comunicación de la Universidad Peruana de Ciencias Aplicadas (UPC). Se entrevistaron solo jóvenes estudiantes universitarias(os) para describir el fenómeno en un sector social en donde se podría pensar, desde algún posible prejuicio de sentido común (tal como que es un comportamiento propio de hombres mayores o de estratos bajos), que no se encontrarían prácticas de ASC. Al entrevistar a una población de cierta homogeneidad, se minimizaría el impacto de las diferencias etarias y sociales en las respuestas de las personas involucradas en la práctica del ASC. Aunque según estudios previos no hay un perfil definido de masculinidad para cometer ASC (Dhillon y Bakaya, 2014), al aumentar las semejanzas entre pares, tenemos una situación más favorable a la posibilidad de conocer el papel que juega la complicidad en "el performance de la masculinidad hegemónica" (Rosales, 2020, p.30), en la continuidad del acoso y en la consideración de este como forma de determinar la supuesta 'cualidad moral' de las mujeres. Por masculinidad hegemónica se entiende aquí los patrones de prácticas y no solo de identidades que permiten la dominación que no se basa en la fuerza sobre las mujeres, y a la masculinidad cómplice como a la de aquellos hombres que reciben los beneficios de aquella sin actuar una versión marcada de ella (Connell y Messerschmidt, 2005).

Al reclutarlos para las entrevistas, se describieron las acciones anteriormente descritas como ASC y se les preguntó si habían actuado de esa manera en alguna oportunidad, comenzando por haber dicho piropos a desconocidas, para luego preguntar "¿qué haces cuando ves en la calle una chica 'bonita' o que te llama la atención?”. Todos los entrevistados habían al menos dicho piropos a desconocidas en primera instancia. Otros admitieron haber llegado a gritar a distancia, decir palabras vulgares, hacer insinuaciones, ruidos de besos, comentarios maliciosos, silbidos, miradas lascivas, o acechamiento. La saturación teórica fue alcanzada cuando en las entrevistas surgía continuamente la figura de la 'mujer buena/respetable' como factor disuasivo del ASC. Para 
completar el estudio, se entrevistaron también a seis chicas adolescentes o jóvenes que hubiesen recibido alguna forma de ASC, por ser la población más expuesta al mismo en Lima, especialmente las jóvenes estudiantes (Vallejo y Rivarola, 2013). El uso de la entrevista como técnica de recolección de datos es apropiada en este estudio porque provee de una perspectiva interna de cómo los sujetos interpretan eventos, instituciones y otros agentes (Weiss, 1994).

El límite de este diseño de investigación es que no permite, ni busca hacer generalizaciones respecto a los perfiles socioeconómicos y sociodemográficos que diverjan de nuestros entrevistados, pues para empezar no se entrevista a grupos comparativos de diferentes estratos sociales o de distintos niveles de educación, ni de diferentes contextos socioculturales. Sin embargo, aunque efectivamente hay diferencias en la concepción de la masculinidad entre estratos sociales en el Perú, por ejemplo, en el mayor énfasis en el vigor y fuerza física en estratos bajos sobre los medios (Fuller, 2018), debe tenerse presente que estudios previos encuentran que no hay diferencias significativas entre estratos en lo que respecta a ciertos elementos que se expresan en el ASC, una concepción que como afirma Callirgos (1996):

fomenta el pasarla bien, desenvolverse con soltura, ser agresivo, 'ser vivo', fuerte, impositivo, macho, etc. El sujeto que cumpla con estos requerimientos recibirá mayor reconocimiento (p.50).

Aunque la práctica del acoso se dirige también hacia personas de sexualidades alternativas, en este trabajo el foco de atención es el acoso que se comete de hombres jóvenes hacia mujeres, principalmente jóvenes o adolescentes, pues es la más prevalente según se ha reportado consistentemente en estudios recientes tanto del Sur como del Norte Global (Sánchez-Díaz, 2019. Nos enfocamos en el ASC que se circunscribe al "machismo cortés" (Rosales, 2020), dejando de lado perpetradores de violencia extrema y los casos como violaciones o golpizas. Las entrevistas se realizaron entre noviembre de 2019 y septiembre de 2020 , por entrevistadores hombres y mujeres también de nivel educativo universitario y de carácter racial diverso. 


\section{El doble enigma teórico del ASC}

Si bien el trasfondo del ASC es una cultura machista, de dominación masculina (Bourdieu, 2000), o de subordinación y objetificación/cosificación de la mujer (Logan, 2015; Molina, López y Chávez, 2020; Quinn, 2002; Sánchez-Díaz, 2019), lo cierto es que tal caracterización requiere de la identificación de mecanismos de mediación entre dicha cultura y algunas de las consecuencias para las víctimas, pues el ASC representa un doble enigma teórico. Primero, de manera general, las mujeres no responden positivamente a un piropo o a la jerga y comunicación usual del ASC. Más aún, en algunos estudios se reporta que los hombres que cometen algún acto de ASC ni siquiera esperan una respuesta de parte de las mujeres (Flores, 2019, p.203). Mientras tanto, la práctica de dar piropos y acosar sigue estando ampliamente generalizada (al menos en la ciudad de Lima, según ya lo reportamos). Si el acoso es $\tan$ poco efectivo en conseguir un fin sexual, e incluso puede que ni siquiera tenga tal finalidad, entonces, ¿por qué sigue siendo tan popular entre los hombres? Más aún, esta pregunta tiene resonancia dado que nos adentramos cada vez más en lo que pareciera una época tendiente a una mayor difusión y rechazo a situaciones perjudiciales para la integridad de las mujeres (si bien incipiente e insuficiente). Así que el primer enigma que representa el acoso callejero es: ¿por qué tantos hombres y con tanta frecuencia aún recurren al mismo a pesar de su baja o nula funcionalidad, más aún en un contexto de creciente oposición por la sociedad en general y por movimientos feministas, una combinación que, al menos hipotéticamente, podría contribuir a desincentivar dicha práctica?

La respuesta que ofrecemos acá está en acuerdo con las tendencias en la literatura sobre el tema, y es que se debe a que se pone en escena un significado particular de la masculinidad para otros hombres, así como para sí mismo: una masculinidad hegemónica y cómplice. En la literatura sobre el ASC, este aspecto en particular ha sido poco considerado en comparación que el papel que tiene el mismo como ejercicio de poder de los hombres sobre las mujeres (Segato, 2016), si bien obviamente estas problemáticas lejos de ser excluyente son complementarias. En efecto, según Logan (2015) puede distinguirse dos categorías de justificación para el ASC, el cual es ante todo una práctica de hombres: conexión con 
otros hombres y control sobre las mujeres. Previos estudios han identificado que, al ser interrogados sobre sus motivos para acosar a las mujeres, los hombres responden cosas como salir del aburrimiento, entretener a otros hombres, o porque es divertido (Benard y Schlaffer, 1996, citados en Dhillon y Bakaya, 2014, 3), por un sentimiento de camaradería juvenil (Benard y Schlaffer, 1984, 71, citados en Logan, 2015, p.204), o por la necesidad de probar la propia masculinidad (Vallejo y Rivarola, 2013). Y aun otros estudios han señalado que la probabilidad de acosar sexualmente aumenta cuando los hombres se encuentran en grupos (Wesselmann y Kelly, 2010). Toda esta evidencia apunta hacia una dimensión grupal, pública y relacional del ASC, confirmada ampliamente en estudios previos (Molina, López y Chávez, 2020).

Pero de seguido, y con ciertas excepciones parciales como Quinn (2002), en la literatura esta tendencia es reducida a la objetificación de las mujeres, o al ejercicio de la dominación masculina o patriarcal a secas (Flores, 2019; Vallone y Quiroga, 2019). Es decir, si bien su significado es derivado del nivel más universal de la subordinación de lo femenino, se pierde un tanto de vista el significado concreto que tiene para los hombres como autoidentificación relacional de género. De hecho, estudios como el de Vallone y Quiroga (2019) señalan la necesidad de profundizar acerca de por qué el ASC es una acción significativa para los hombres, comolegitimación entre pares. En otras palabras: el ASC puede ser concebido como una puesta en escena, una representación de la masculinidad con consecuencias no solo para las víctimas sino también para los victimarios (aunque obviamente de distinta naturaleza).

Pero hay un segundo aspecto o enigma mucho menos considerado en estudios previos, a saber, el de los límites que la presencia de mujeres implican para el ASC. En efecto, nuevamente bajando del nivel de análisis de la significación universal de la subordinación femenina y yendo hacia un aspecto más particular de la cultura, o que en todo caso habría que comprobar o contrastar con estudios en otros contextos, en el caso que estudiamos los hombres jóvenes sienten una coacción a no realizar ASC cuando están en presencia de otras mujeres que son significativas para ellos. Si es así, entonces la relación entre la cultura llamada patriarcal y el ASC no es lineal: necesitamos alguna otra mediación para entenderla. La universal subordinación de género no explica directamente este poder disuasivo. Aquí propondremos que 
esta consideración se puede entender porque proviene de la concepción no intencional del ASC también como una forma de 'determinar' el supuesto carácter moral de las víctimas para los hombres que cometen ASC, dividiendo el espacio simbólico entre distintos tipos de mujeres: las merecedoras de respeto y las que padecen alguna deficiencia moral y, por tanto, no son dignas de respeto. Por ello, el ASC no es aceptable delante de las mujeres "buenas", mientras las respuestas que las mujeres desconocidas dan al acoso hacen público su carácter moral. A continuación, propondremos una interpretación para incorporar los tres aspectos en un mismo marco consistente: la subordinación de lo femenino a la dominación masculina, la puesta en escena de la masculinidad ante otros hombres junto con las consecuencias de ello para el individuo social, y el rol simbólico de la mujer en la limitación (al menos parcial) al ASC. Estos tres niveles conducen a concebir, relacionalmente, el ASC también como una forma de 'determinar' la 'cualidad moral' de las mujeres según las respuestas que estas den al acoso.

\section{El marco general: el ASC y la dominación masculina}

Una cultura machista o de dominación masculina ha sido ampliamente señalada como la principal causante del ASC. Estudios previos afirman que el ASC tiene como función reproducir las jerarquías de género (Castro y Vázquez, 2008, citado por Vallone y Quiroga, 2019, p.3). En general, múltiples estudios conciben el ASC como una cuestión de poder basado en el género (Chafai, 2020; Vallejo y Rivarola, 2013). Puede entonces decirse que es una situación marcada por la dominación masculina. Bourdieu (2000) ha hablado de la dominación masculina como un caso de violencia simbólica,

(...) violencia amortiguada, insensible, e invisible para sus propias víctimas, que se ejerce esencialmente a través de los caminos puramente simbólicos de la comunicación y del conocimiento o, más exactamente, del desconocimiento, del reconocimiento o, en último término, del sentimiento (p.12)

En esta, las mujeres son convertidas en objetos simbólicos de intercambio (Bourdieu 2000), en la economía de los bienes simbólico en un espacio público aún dominado ampliamente por los hombres (p.117). Y particularmente el acoso sexual en general es concebido como tendiente a la 
posesión sin más, la afirmación de la dominación en estado puro (Bourdieu, 2000, p.35). Puede verse como parte de la agorafobia socialmente impuesta que conduce a las mujeres a excluirse 'voluntariamente' del ágora (Bourdieu, 2000, p.54). Segato (2003) coincide con esta visión al menos en un sentido, a saber, que la dominación masculina se da como una naturalización de la anatomía proyectada en lo simbólico, lo cual de vuelta se emplea para justificar una jerarquía de los géneros-Segato (2003, p.243) le llama la ideología de la coacción anatómica' occidental. En sus rasgos generales, esta práctica forma parte de la universalidad de la subordinación femenina, que es resultado de la identificación cultural de la mujer con el trasvase o la intermediación entre naturaleza y cultura, por un lado, y por otro lado del hombre con la cultura y sus actividades superiores (Ortner, 1979), y estas vinculadas al espacio público. En este marco general se encuadra el significado del ASC, consistente con la demostración publica de la hombría de los varones (Callirgos, 1996). Nosotros añadimos: $\mathrm{y}$ de rebote, también con la demostración pública de la moral de las mujeres. Veamos ahora su desenvolvimiento en nuestro caso específico.

\section{Entre la cultura y el sí-mismo de la masculinidad hegemónica: la puesta en escena del ASC}

Estudios previos encuentran que elevados niveles de ASC se dan en lugares con mucha presencia de otras personas y a plena luz del día (Dhillon y Bakaya, 2014). Así mismo, estudios previos sobre el ASC en Latinoamérica encuentran que a mayor sexismo (no solo por la masculinidad definida por criterios estereotípicos), mayor atribución de culpabilidad a la víctima y menor percepción de la gravedad del acto (Angelucci et al, 2020). Más aún, en la ciudad de Lima, no hay zonas ni horarios específicos en donde ocurra el ASC, excepto por una característica: hay más casos en donde hay una concentración de hombres, tales como paraderos de taxis, construcciones, zonas en donde se realizan obras públicas, venta de productos para autos, ferreterías, o estadios (Vallejo y Rivarola, 2013). Y aun otros estudios en el contexto estadounidense muestran que 'mirar a las chicas' funciona como un juego que los hombres juegan para construir una identidad masculina compartida, y del que esperan que todos sepan que lo están haciendo (Quinn, 2002). Estos hallazgos son fundamentales 
para nuestro argumento, porque implican que en el ASC hay una concepción de la masculinidad según la cual la misma se ha de exponer a luz del día y ante el público. En otras palabras: la misma requiere de una puesta en escena, para sí mismos y para otros hombres.

Por lo cual, si bien el ASC ocurre en el marco de una cultura machista que define roles tradicionales de género, en donde se tiende a culpar a la mujer por el ASC y en donde se justifica la violencia como forma de ejercer autoridad (Angelucci et al, 2020), dicha cultura pasa por el aspecto público de la estructuración de los códigos de dicha masculinidad que necesita acosar a las mujeres: el contexto mismo y la actuación de los códigos, los cuales son estructurantes de la cultura desde un punto de vista pragmático. Es decir, va más allá de que no se evita cometer el ASC en público y a la vista de todos, sino que incluso se considera como una oportunidad (Dhillon y Bakaya, 2014): la oportunidad de poner en juego un significado para otros hombres,y, según argumentaremos más adelante, de exponer al público (masculino, principalmente) las respuestas de las mujeres al ASC. Esto quiere decir que la presencia de esos otros hombres es más que relevante para el significado del ASC: es una masculinidad que necesita ser probada y de testigos que la validen (Callirgos, 1996; Rosales, 2020). Así lo confirman nuestros entrevistados:

(...) más que todo lo hago con mis 'patas' [amigos] porque es un 'cochineo' hacia ellas, un morbo quizás que tenemos y ya (...) depende de dónde me encuentre como que de una vereda a otra 'mas-o' [más o menos]; o en un paradero, puede que ella este ahí y ya yo con mis 'patas' [amigos] en una esquina ahí silbamos, le coqueteamos (...) (P: ¿De dónde nace eso de coquetear de esa forma?) R: lo fui aprendiendo de mi grupo de amigos. Básicamente como un juego ( $\mathrm{P}$ : ¿ Has visto ese tipo de cosas en tu familia quizás?) R: Claro, a mis tíos y primos, aunque ellos son peores, ¿ah? (P: ¿Cómo eran?) R: Mi tío me contaba que con sus amigos él también salía a piropear a veces, así en 'mancha' (en grupo). Y como que mi primo también hacía lo mismo y yo a veces andaba con él, entonces ya se me fue quedando. (P: ¿Y en casa?). R: A veces mi hermano mayor (entrevistado 5.)

En mi grupo de amigos siempre nos pasamos la voz cuando una chica guapa pasa por nuestro camino, en la calle, en el cine, en la Universidad (entrevistado 10). 
(...) mis amigos son los que principalmente me influenciaron eso (entrevistado 11).

Vallone y Quiroga (2019) han sugerido que podría tratarse de un tipo de ritual que posiciona a los hombres frente a sus pares, mientras que los diferencia de las mujeres. Serían “... auténticas pruebas de virilidad orientadas hacia el reforzamiento de las solidaridades viriles”, una visión que hace aparecer a la virilidad como noción relacional (Bourdieu, 2000, pp.70-71).

No soy de piropear solo soy de mirar, pero bueno a veces si estoy en mi barrio o en lugares donde puedo dármela de 'bacancito' (valiente / altanero) o estoy con mis 'patas' (amigos), nunca falta un silbido, se podría decir así (entrevistado 12).

Esto es consistente con estudios previos que muestran que el sentido de anonimato que ofrece el estar en grupo $y$, especialmente, la idea de establecer vínculos grupales, favorecen la proclividad de ciertos hombres al ASC (Gardner, 1995; Wesselman y Kelly, 2010). Por último, consistente con previos estudios (Molina, López y Chávez, 2020) la lectura de la propia cultura como 'machista' se convierte en justificación para el ASC:

Bueno estamos en Perú hermano, tú sabes que aquí en Perú el machismo abunda demasiado y lo que importa es lo que la mayoría piensa, que los hombres son unos machistas y bueno en realidad es muy cierto y creo que la mayoría reaccionaría como yo (entrevistado 12).

Sin embargo, como un posible tema para desarrollar en otro estudio, también se nota a partir de las entrevistas que existe una ambivalencia entre los hombres que practican el ASC, pues para algunos se establecen ciertos límites que no deben ser transgredidos, como por ejemplo comentarios sexuales explícitos, tocamientos, frotamientos, exhibicionismo y masturbación pública, los cuales son catalogados como resultado de enfermedades mentales o síntomas de problemas psicológicos (Vallejo y Rivarola, 2013). Es decir, entre quienes practican el ASC, hay una subdivisión entre lo aceptable y lo no aceptable que valdría la pena desarrollar en otra investigación (también Flores [2019], Gaytán [2007], y Quinn [2002] notan esta distinción que hacen los mismos hombres entre tipos de piropos o de 
actos supuestamente "halagadores" hacia las mujeres, y actos "enfermizos") y que podría ser parte de una forma de "machismo cortés" (Rosales, 2020):

O sea, tipo yo siempre he sido respetuoso, pero siempre hay uno que quiere ser el escandaloso o el extremista (entrevistado 8).

\section{Las y los individuos sociales y el ASC}

El ASC es resultado de una sexuación del espacio social (Martín, 2014), dividido entre el espacio público para los hombres y el espacio privado (o doméstico) para las mujeres (Ortner, 1979). Pero dicha sexuación tiene resonancia sobre el espacio de lo individual. En efecto, el control masculino del territorio público cambia los hábitos de las mujeres, les conduce a tomar medidas preventivas tales como evadir áreas de la ciudad, no salir solas, o quedarse en casa, además de que influencia el cómo vestir (Alcalde, 2020; Martín, 2014). La responsabilidad de evitar agresiones sexuales recaería así sobre las propias mujeres, pues se asume a los hombres como 'incapaces de controlarse', regidos por una norma que dicta que les es imposible dominar los impulsos sexuales (Flores, 2019; Molina, López y Chávez, 2020; Quinn, 2002). Esto también se constata en el caso de la ciudad de Lima (Fuller, 2005). Mientras, se les enseña a las chicas cómo vestir para evitar la atención masculina, evitar ciertos lugares, o no hablar de manera que se les vea como sujetos de deseo (Vallejo y Rivarola, 2013):

(...)desde pequeña siempre me repetían que debía cuidarme porque había mucha gente mala y que a veces los hombres les gustaba aprovecharse de una, que cuide como me visto porque a veces tu ropa puede decir mucho de ti (entrevistada 2).

(...) no salía mucho, paraba con mis primos mayores y mi hermano, jugaban conmigo y yo pues me quedaba ahí en mi casa, muy difícil que salga a jugar con amigos de mi barrio (entrevistada 5).

Una consecuencia de que se expulse a las mujeres de los espacios públicos, y sobre todo de ciertos lugares y a ciertas horas, es que la única estrategia considerada por las mujeres realmente eficiente para salir a dichos espacios públicos acompañadas con un hombre (Dhillon y Bakaya, 2014; Vallejo y Rivarola, 2013), o por asociación con un hombre conocido 
aun si este no está presente (Vallone y Quiroga, 2019). El resultado es un reforzamiento de una relación desigual hombres-mujeres en la apropiación cotidiana de lo público. El responder por sí mismas, ha sido resultado de un proceso de trabajosa reconfiguración del sí-mismo:

(...) las últimas veces que me han tirado un piropo he logrado contestar, cosa que no pasaba antes. Evidentemente no reacciono de una manera buena. (P: ¿Por qué antes no respondías?). R: porque lo había normalizado, o sea que un extraño me lance un piropo, me lance alguna mirada o que me hostigue, $y$ nadie hacía nada al respecto, entonces yo tampoco me sentía obligada a hacer algo. Pero recién todo esto se ha empezado a cuestionar más, entonces yo también me he empezado a cuestionar, y eso me ha llevado a ya no quedarme callada y responder a ese tipo de personas. ( $\mathrm{P}$ : ¿de qué manera respondes?). R: siempre es de mala manera porque exploto cuando me hacen algún comentario de ese estilo, porque no sé, desde que tengo 12 años aproximadamente me he topado con ese tipo de comentarios y ya jode, ¿no? (entrevistada 1).

Ok... mi comportamiento frente a un acoso, en algún... bueno, antes me pasaba como que eran bajos, ¿entiendes? Como que te explico, me quedaba callada, y pues, es más, hasta todavía me han acosado físicamente, también verbalmente, entonces... me han mirado, me han tocado, bueno... obviamente antes no, cuando..., también bueno..., bueno también están..., bueno..., también meten ahí los roces, cosas así, ¿verdad? Entonces como te explico, antes sí era demasiado bajo, ¿sabes? Pero creo que era todo porque tenía baja autoestima, y pues..., ahora respondo (entrevistada 4).

Además, como confirman otros estudios, cuando la respuesta de la víctima es la confrontación, el acosador detiene el acto, mientras ignorar o mostrar sumisión refuerza la conducta de acoso (Molina, López y Chávez, 2020). Respecto a la vestimenta, en algunos casos sí puede llegar a llamar la atención de un acosador:

Mientras más apretado o ceñido mejor, creo. Uhm, la ropa creo que dice mucho de una persona, si ellas visten así, en mi opinión es que quieren ser piropeadas, ¿no? Ellas quieren ser vistas sí o sí. (P: ¿No crees que simplemente es así, porque les gusta y ya?) ... R: Yo creo que hay algo más a fondo de eso, o bueno he conocido chicas que me han confesado que se vestían así para llamar la atención de sus 'crush' (entrevistado 9). 
Sin embargo, como en estudios previos (Dhillon y Bakaya, 2014; Gaytán, 2007), también las mujeres jóvenes entrevistadas afirmaron que la ropa no es determinante para recibir ASC (más abajo volveremos sobre este punto):

Igual yo no creo en eso, a veces salgo en pijama, así toda sucia y aun así me han piropeado, así que no creo que la ropa defina algo (entrevistada 2).

Yo creo que no [es determinante la ropa] porque hasta cuando salgo con pijama me molestan (entrevistada 5).

Yo salí a la calle completamente tapada con la mascarilla y el traje protector, supongo que mi cuerpo se podía notar, mi silueta. Bueno, la cosa es que a mí y a mi mama nos dijeron 'que ricas están' y eso me sorprendió demasiado porque dicen que a nosotras las mujeres nos molestan por el tipo de ropa que utilizamos, pero en este caso no fue así. Entonces, me confundió demasiado. Si estoy en verano me molestan y si es invierno igual, hasta en pandemia (entrevistada 6).

Esto lo confirmaron algunos de nuestros entrevistados:

(P: ¿Qué interpretas de su ropa?) No, ninguna. No, no me afecta su ropa. No es una variante (entrevistado 7).

No, no me fijo en nada [relativo a la ropa]. Eh... porque me agrada. Nada, solo me gusta su aspecto físico (entrevistado 8)

Realmente, el dominio masculino del espacio público puede llegar a doblegar o a inspirar un retraimiento del símismo de las mujeres:

Me siento intimidada, me da bastante miedo, me siento inferior hacia esa persona. Con tan solo me digan algo de esa índole me pongo a llorar. Ocurrió más cuando comencé a andar sola a la edad de trece años. Mayormente cuando estoy pasando por construcciones, los albañiles molestan y, o sea, sé que la mayoría de veces pasa y no se dedican a seguirte. Entonces solo les digo insultos para que se callen. Les digo en plan 'apágate concha e' tu madre' y ese tipo de cosas. Con los piropos lindos, a veces agradezco o me quedo callada. Con los toques mayormente me pasan en el transporte público, pero no le digo nada a mi acompañante hasta llegar a mi casa. Cuando sucede eso realmente me afecta, hasta me hace dudar mucho de mi personalidad ese tipo de situaciones, porque bueno soy muy coqueta siempre paro de sonrisa de 
oreja a oreja y tengo miedo de que las cosas lleguen a malinterpretar mi personalidad (entrevistada 6).

Es así como previos estudios han llegado a considerar el ASC como terrorismo sexual, al recordar permanentemente a las mujeres su vulnerabilidad en los lugares públicos (Flores, 2019; Sánchez-Díaz, 2019). Mientras tanto, desde el punto de vista del sí-mismo masculino el efecto es opuesto, es el de una autoafirmación o autovalidación:

Cuando la veo gano esa satisfacción que hace un momento no tenía. Me encanta hacerlo porque me hace sentir más hombre. Cosas así ("risas"). En cierto modo, voltear y mirarla me hace sentir varón o que puedo lograr muchas cosas. Me siento lo máximo. Pero sin duda me siento satisfecho. Es algo que uno no puede contar o describir con palabras. Pero es una sensación que te deja satisfecho (entrevistado 15).

\section{La presencia de las "buenas mujeres" como elemento disuasorio del ASC}

Estudios previos encuentran que de manera general el ASC se da cuando se percibe a las mujeres como vulnerables, inferiores a los hombres y con menos derechos (Cortázar 2019, citado por Molina, López y Chávez, 2020, p.377). Sin embargo, hay una situación en la cual la presencia de mujeres disuade a los hombres del ASC y, dada la subordinación universal de lo femenino, antes expuesta, esto se convierte en un factor que hay que explicar. A nuestros entrevistados se les interrogó bajo cuáles circunstancias no acosarían o darían un piropo a las mujeres en la calle. Los entrevistados respondieron que la presencia de otra mujer conocida o de una familiar mujer era un factor determinante. Hasta donde alcanza nuestro conocimiento, solamente un estudio previo consideró este factor, aunque de manera tangencial (Wesselman y Kelly, 2010):

Ahh... cuando estoy cerca de mi mamá, o sea ahí se merece más respeto pues... o con una amiga que yo sepa que no es muy fan de los piropos, o cuando estoy con mi flaca tampoco lo hago, ahí como que me contengo cuando hay mujeres de mi interés cerca mío no suelo hacer eso (entrevistado 2).

Cuando estoy en la U [universidad] me entra cierta vergüenza, siento que me pueden acusar o que algún profesor me oiga y me llame la atención... También, 
cuando está presente mi mamá (entrevistado 9).

Recuerdo que siempre nos 'palteabamos' (avergonzábamos) cuando había una flaca (una chica) que conocíamos, ponte (supón) una amiga o ex, solíamos controlarnos ("risas"), hasta ahora creo que es igual, o sea cuando ando acompañado... no suelo voltear. (P: ¿con tu familia?). R: jah! pues tampoco (entrevistado 11).

Es decir, si hay mujeres cercanas, su presencia tiene un efecto disuasivo sobre el ASC. Esto puede entenderse, primero, como una confirmación de la importancia que el ASC tiene ante otros hombres a lo cual Quinn (2002) caracteriza como un factor homo-social y al que ya nos hemos referido. Pero esto tiene una segunda implicancia: el ASC funcionó en la práctica para determinar la moralidad de las mujeres de acuerdo con sus respuestas al acoso, lo cual presupone una masculinidad que se coloca en la posición de juez. Por ejemplo, cuando la acosada es cercana:

( $\mathrm{P}:$ ¿Y alguna vez tu hermana ha señalado que la han piropeado o algo por el estilo?). R: sí... un par de veces, inclusive había un 'pata' [aquí en el sentido de 'un tipo'] que medio la acosaba. (P: ¿y tú cómo reaccionaste a eso?). R: Pues me llegó, porque era mi hermana, y quise ir a hablar con el 'pata' ['el tipo'] a ver porqué hacía eso, pero mi hermana no me dejo y dijo que ella lo solucionaría (entrevistado 11).

El hecho de que sujetos que con regularidad hayan cometido ASC, se restrinjan de hacerlo frente a otras mujeres nos conduce a una última consideración: la distinción entre las 'buenas' y las 'malas' mujeres en el espacio público que, al menos en Lima, es "Lugar de jerarquías y conflictos de género” (Rosales, 2020, p.20).

\section{El ASC y la determinación de la moral pública de las mujeres}

A los entrevistados se les interrogó qué pensaban sobre las reacciones de las mujeres acosadas (sin usar este término). Dos tipos de respuestas destacaron. Por un lado, hay una concepción positiva de la mujer que rechaza a los piropos o avances sexuales de extraños y una concepción negativa de quienes los aceptan: 
Yo creo que estas chicas responden así (positivamente) porque creo que le faltara (SIC) atención en casa y con ese tipo de piropos yo les doy esa atención. (P: ¿Por qué no todas lo hacen?) R: Supongo que algunas son más de casa ( $\mathrm{P}:$ ¿a qué te refieres con eso?). R: A que tuvieron una buena educación, son respetuosas, pero hay otras que quizás le falta una buena imagen materna o paterna y por eso como que buscan esa atención (entrevistado 5).

(...) me imagino que a algunas les debe incomodar, pero también hay otras a las que sí les gusta ( $\mathrm{P}$ : ¿por qué dices eso?) R: Porque las hará sentir más bonitas o apreciadas... además hay algunas que se jactan de eso (P: ¿Qué las piropean?). R: sí, yo conozco una amiga que siempre decía que la piropiaban, pero no lo decía como algo malo, así que asumo que a algunas les gustará (...) me imagino que las que responden positivo son las 'bellaquitas'. (P: ¿bellaquitas?). R: sí ["risas"], las chibolas [jovencitas] que van a tonos [fiestas] y les gusta 'perrear' con extraños. Me imagino que son así porque buscan atención (...) la mayoría son decentes, o sea se hacen respetar, porque creo que una 'flaca' [una chica] que sale de fiesta a cada rato y se mete con varios extraños no tiene una educación adecuada (entrevistado 11).

En este caso, el ASC determina o identifica a la 'buena mujer' en un sentido negativo, pues una respuesta favorable al llamado del hombre significa un déficit moral del sujeto femenino, ya sea porque le falta una 'buena educación', ya sea porque no ha recibido suficiente atención parental. La respuesta negativa a los piropos es interpretada como un signo moral de la mujer también en otro sentido:

(P: ¿Tienes hermanas?). R: Sí dos. (P: ¿A ellas le gustan que las piropeen?). R: A una sí, la otra los manda a la mierda ("risas") (P: ¿por qué crees que sea eso?). R: Porque ella tiene otra crianza, una vez una pata le dijo: 'Hola mi amor, qué estás haciendo, vamos por un par de cervezas'. Ella agarró y saco un balde de agua de su casa y lo mojó. Le dijo: Viejo de mierda, deja de pretenderme'. Esas son las mujeres que se saben dar a respetar, porque si la mujer no hace nada ese pata [aquí en el sentido de 'ese tipo'] puede contarle a otro y así de boca en boca, hasta que la reputación de la chica se arruine. Entonces, ¿tú como quedas como mujer...? (entrevistado 14).

En este caso, la mujer sabe cuidar su reputación como 'buena mujer', si no responde favorablemente a una invitación o llamado sexual público. Es decir, la respuesta 
negativa a un avance sexual o a un piropo denota que la chica es evaluada y ha pasado la prueba, una prueba que es recurrente. De lo contrario, arruina su 'reputación' delante de otros hombres. Por lo tanto, un efecto no intencional de esta práctica es que también se fuerza a "poner en escena”, a mostrar públicamente, la respuesta de las mujeres al mismo y así determina la composición moral de estas. Dice otro entrevistado al respecto:

Siempre he respetado a mis 'flacas' (novias) porque las veo distintas a las chicas con las que 'gileo' (coqueteo) o intento piropearlas. Ellas son otro lote; en cambio mis novias son unas damas (entrevistado 15).

Hay un tercer elemento en esta dirección: el uso de la vestimenta también se lee como un signo de la personalidad de las mujeres, lo cual complejiza el rol de la misma en el ASC. Pues si bien, como lo hemos visto anteriormente, no es un factor determinante para favorecer o desfavorecer el acoso, sí se emplea como un indicador para 'determinar' la constitución moral de las mujeres. Por ejemplo, respecto a la autoconfianza de las chicas:

La ropa que es pegada o cosas así, no sé... les resalta más el cuerpo. Estoy totalmente a favor de ese tipo de ropa ("risas")... Yo pienso que las que usan ese tipo de ropa son seguras de sí mismas y que no tienen complejos, aparte debe ser porque les gusta su cuerpo, ¿no? (Entrevistado 10).

Pero, por otra parte, más en general y de manera paradójica, los entrevistados piensan que las chicas quieren ser 'piropeadas', o deberían quererlo:

(P: ¿Crees que tu piropo puede llegar a incomodarla?) R: No, no creo. Es más, debe sentirse bien y subirse la autoestima. (P: ¿Por qué?). R: Es que notaron que está guapa, la verdad no entiendo como algunas miran mal, solo deben sonreír. Algunas reaccionan bien, supongo que lo entienden (Entrevistado 9).

Lo hago más para molestarlas, no tengo ningún fin que sea el de 'pasarme de la raya'. (P: ¿A qué te refieres?). R: Hacer daño físico o por último violarlas. Nada que ver. Algunas se exaltan por 'huevadas' [tonterías]. Además, no tiene nada de malo decirle lo buena, bonita que está (entrevistado 10). 
Así que, por lo tanto, para añadir a esta complejidad, la mujer debe usar ropa 'ajustada' para mostrar su autoconfianza y llamar la atención de los hombres, pero no aceptar los avances de extraños pues denotaría una falta moral, aun cuando debe considerar dichos avances como algo positivo respecto a ella misma. De esto resulta que el ASC es entonces también una suerte de forma de determinar el carácter moral de las mujeres, en un doble sentido. Por una parte, ellas deben querer la atención masculina, pues es un reconocimiento a su atractivo físico. Pero, por otra parte, no deben reaccionar positivamente a dicha atención, pues eso mostraría que tienen un 'desperfecto' en su constitución como sujetos morales, expuesta al público (masculino). Lo cual quedaría evidenciado en su 'liberalidad' traducida, en el mejor de los casos, como falta de autoestima.

\section{Conclusiones}

Concebir al ASC como otra forma de violencia simbólica contra la mujer se inscribe en un reclamo de especialización o delimitación de los espacios sociales, en donde ya no aparece la oposición calle (púbico) / casa (privado), como espacios sexuados: la sexuación quedaría así limitada a un espacio propio y decidido por los sujetos, en lugar de ser el espacio potencialmente agresivo y enajenante que de hecho resulta para las mujeres. En este trabajo hemos argumentado que, en el marco general del fenómeno de la dominación masculina como masculinidad hegemónica, el ASC representa un doble enigma: primero su falta de objetivos instrumentales (sexuales) inmediatos y segundo la negativa a llevarlo a cabo delante de ciertas otras mujeres. La respuesta a estos enigmas es relacional en tres facetas. El primer aspecto nos ha llevado a coincidir con estudios previos de diversos contextos sobre el hecho de que el factor primordial del ASC es homo-social, es decir, es un performance que adquiere pleno sentido si se lleva a cabo para otros hombres y para sí mismo como hombre, para mostrar la pertenencia al grupo dominante y como prueba de dicha pertenencia. Mientras tanto, el segundo punto nos ha conducido a argumentar que el ASC funciona también como una práctica para determinar la cualidad moral de las mujeres, al menos en el contexto aquí estudiado. Esto surge de la observación de que la presencia de ciertas mujeres disuade a los hombres de llevar adelante 
el ASC, pero que el solo marco de la dominación masculina universal, y la correlativa cultura machista, no nos explicaría este hecho de manera directa. Hemos desarrollado por ello una explicación coherente con dicho marco, pero haciendo notar la particularidad de esta observación. Para esto, indagamos acerca de lo que los jóvenes entrevistados pensaban sobre las mujeres acosadas. El resultado fue que el ASC es usado de manera derivada por los hombres también como una forma de evaluar a las mujeres como sujetos morales, para distinguir las 'buenas' de las 'malas' mujeres. Y esto quedaría expuesto públicamente en las respuestas de dichas mujeres al ASC: deben sentirse bien por el mismo como reconocimiento masculino, pero no responder positivamente. Quizá los límites sean menos difíciles de transgredir para hombres que sean capaces de violar a una mujer o de darle una golpiza ("machismo brutal"), pero al menos puede pensarse que en muchos casos la presencia de otras mujeres significativas para los hombres puede en efecto disuadir esta práctica, si bien de manera circunstancial y no definitiva, pues es una disuasión correlativa a la lógica cultural de la dominación masculina. Este es un hallazgo basado en un caso que habría que comparar con estudios en otros contextos.

\section{Referencias bibliográficas}

AlCALDE, María Cristina (2020). Gender, autonomy and return migration: negotiating street harassment in Lima, Peru. En Global Networks, volumen 20, Nº 1 , pp. 25-41.

Angelucci, Luisa; Romero, Arturo; Marcano, Thaimí; Aquino, Sofía; Carrero, Ana Paula; De Jesús, Rossmary; Tapia, Vanessa (2020). Influencia del sexismo, el rol sexual y el sexo sobre percepción del acoso callejero. En Revista Investigium IRE: Ciencias Sociales y Humanas, volumen XI, $\mathrm{N}^{\circ} 1$, pp. 28-45.

Bourdieu, Pierre (2000). La dominación masculina. Barcelona: Anagrama.

Callirgos, Juan Carlos (1996). Sobre héroes y batallas. Los caminos de la identidad Masculina. Lima: Escuela para el Desarrollo-DEMOS.

Chafal, Habiba (2020). Everyday gendered violence: women's experiences of and discourses on street sexual harassment in Morocco. En The Journal of North African Studies, pp. 1-20. Versión en línea: https://doi.org/10.108 
O/13629387.2020.1743184

Connell, R.W y Messerschmidt, James (2005). Hegemonic Masculinity: Rethinking the Concept. En Gender \& Society, volumen 19, N6, pp. 829-859.

Davidson, M. Meghan; Butchko, Michael; Robbins, Krista; SHerd, Lindsey; Gervais, Sarah (2016). The Mediating Role of Perceived Safety on Street Harassment and Anxiety. En Psychology of Violence, volumen 6, $\mathrm{N}^{\circ} 4, \mathrm{pp}$. 553-561.

Dhillon, Megha; Baraya, Suparna (2014). Street Harassment: A Qualitative Study of the Experiences of Young Women in Delhi. En SAGE Open, volumen 4, N³, pp. 1-11.

Di Leonardo, Micaela (1981). Political Economy of Street Harassment. En Aegis. Magazine on Ending Violence Against Women, pp. 51-57.

Flores, Marieliv (2019). Género y espacio público: El acoso sexual callejero como muestra de hombría. En Hernández, W. (ed.), Violencias contra las mujeres, la necesidad de un doble plural (pp. 183-210). Lima: GRADE.

Fraser, Erika; KalPabA, Viswanath; MAClEAN, Laura (2017). Violence against Women and Girls, Infraestructure and Cities (Briefing Paper). En ICED-Infraestructure \& Cities for Economic Development, pp.1-75.

Fuller, Norma (2005). Cambios y permanencias en las relaciones de género en el Perú. En VALDÉs, T. y VALDÉs, X. (eds), Familia y vida privada (pp.107-129). Santiago: FLACSO-Chile/CEDEM/UNFPA.

Fuller, Norma (2018). El cuerpo masculino como alegoría y como arena de disputa del orden social y de los géneros. En Fuller, N. (ed.), Dificil ser hombre. Nuevas masculinidades latinoamericanas (pp.25-45). Lima: PUCP.

Gardner, Carol (1995). Passing By: Gender and Public Harassment. Berkeley \& Los Angeles: University of California Press.

GAYTÁn, Patricia (2007). El acoso sexual en lugares públicos: un estudio desde la Grounded Theory. En El Cotidiano, México, volumen 22, $\mathrm{N}^{\circ}$ 143, pp. 5-17.

Logan, Laura (2015). Street Harassment: Current and Promising Avenues for Researchers and Activists. En Sociology Compass, volumen 9, $\mathrm{N}^{\circ} 3$, pp. 196-211.

Martín, María (2014). El Espacio Público como Territorio Sexuado: El caso del Acoso Callejero desde un Enfoque de Género. En Revista El Topo, Valparaíso, N 3, pp. 88101. 
Martínez, Miriam (2018). Acoso Sexual Callejero como forma de violencia de género y experiencia piloto en población femenina de la Universitat de les Illes Balears. Tesis sin publicar, Facultat de Psicología, Universitat de les Illes Balears, Islas Baleares.

Molina, Nancy; López, Sergio; Chávez, Guillermina (2019). La dinámica del acoso sexual callejero desde el discurso de hombres jóvenes. En MILLCAYAC-Revista Digital de Ciencias Sociales, volumen 6, N 11, pp. 363-390.

ORTNER, Sherry (1979). ¿Es la mujer con respecto al hombre lo que la naturaleza con respecto a la cultura?. En Harris, O. y Young, K. (comps.). Antropología y feminismo (pp. 109-131). Barcelona: Anagrama.

Quinn, Beth (2002). Sexual Harassment and Masculinity: The Power and Meaning of 'Girl Watching'. En Gender 8 Society, volumen 16, $\mathrm{N}^{\circ}$ 3, pp. 386-402.

Rodríguez, Paula (2020). Acoso sexual callejero: un análisis criminológico y con perspectiva de género. Tesis sin publicar, Grado de Criminología en la Universidad Autónoma de Barcelona, Barcelona.

Rosales, Raúl (2020). Masculinidades en movimiento. Desde la vida cotidiana hasta las políticas públicas. Lima: Vivirsinenterarse

Segato, Rita (2003). Las estructuras elementales de la violencia. Ensayos sobre género entre la antropología y el psicoanálisis y los derechos humanos. Buenos Aires: Universidad Nacional de Quilmes.

Segato, Rita (2016). La guerra contra las mujeres. Madrid: Traficante de sueños.

SÁnchez-DíAz, Lucía. (2019). Street harassment perception and its relations with self-objectification of women. En Interdisciplina, volumen $7, \mathrm{~N}^{\circ} 17, \mathrm{pp}$. 153-170.

Vallejo, Elizabeth y Rivarola, María (2013). La violencia invisible: acoso sexual callejero en Lima Metropolitana y Callao. En Serie Cuadernos de Investigación IOP, $\mathrm{N}^{\circ} 4$, pp.1-19. Lima: PUCP - Instituto de Opinión Pública.

Vallone, Florencia y Quiroga, Paula (2019). Violencia de género: una tipificación del acoso callejero. Ponencia presentada en las XIII Jornadas de Sociología (pp. 1-15) Facultad de Ciencias Sociales. Universidad de Buenos Aires, Buenos Aires.

WeIss, Robert (1994). Learning from Strangers. The Art and Method of Qualitative Interview Studies. New York: The Free 
DETERMINANDO LA “MORALIDAD PÚBLICA" DE LAS MUJERES. UNA PERSPECTIVA RELACIONAL SOBRE EL ACOSO SEXUAL CALLEJERO EN ESTUdiantes UNiversitarios DE LiMA, PERÚ

Press.

Wesselmann, Eric; Kelly, Janice (2010). Cat-Calls and Culpability: Investigating the Frequency and Functions of Stranger Harassment. En Sex Roles, No 63, pp. 451-562.

Fecha de recepción: 10 de octubre de 2020 Fecha de aceptación: 2 de febrero de 2021

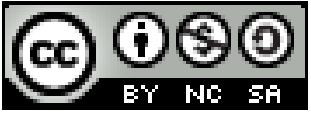

Licencia Creative Commons Atribución-No Comercial-Compartir Igual 4.0 Internacional 
